Hautwunde nach 4-6 Monaten der Festigkeit des benachbarten natürlichen Gewebes.

Die Festigkeit der chirurgischen Naht selbst wird wesentlich beeinflußt von der Anzahl der haltenden Einzelfäden pro Längeneinheit der Wunde, ferner von der Dicke des Fadens, der das Gewebe schneidend beansprucht, und schlieBlich ganz wesentlich von der Menge des mit der Naht gefaßten Gewebes. Diese Faktoren lassen sich durch in vitro-Versuche kurvenmäßig belegen.

Im Tierversuch wird schließlich demonstriert, daß sehr fest angezogene Fäden zur Nekrose des gefaßten Gewebes und damit zur Schwächung der Naht besonders in der 2. Woche der Wundheilung führen. Mit Hilfe der Infrarotphotographie kann die Drosselung der Blutzufuhr im direkten Narbenbereich bei zu eng gelegten oder zu fest angezogenen Fäden in vivo gezeigt werden.

Die Kalkulation der an der Naht angreifenden Kräfte vermag in Korrelation mit den gewonnenen Daten über die Nahtfestigkeit Hinweise für die Erkennung derjenigen Patienten zu geben, die zur Verhütung einer Wundruptur in den Genuß spezieller Nahtmethoden kommen sollten.

\title{
101. Analyse der postoperativen Letalität nach Laparotomien
}

\section{BÜRGER-Erlangen}

\section{Analysis of Postoperative Mortality after Laparotomy}

Summary. After 11519 laparotomies 668 patients died $(5.6 \%)$ while still in hospital. The peak after first operations occurred about the 5 th day, after second operations on the 2nd day and after third operations on the 1st day. Autopsies were performed on 450 patients. The most frequent surgical cause of death was peritonitis (156 patients) which however had been present preoperatively in one third of the cases. 42 patients died of haemorrhage and 3 of postoperative ileus. $55 \%$ died of non-surgical complications. A comparison with the risk factors found, such as old age, poor ECG, diabetes and obesity, shows that only $4.9 \%$ of all fatal cases were free from these preoperatively known risk factors.

Zusammenfassung. Nach 11519 Laparotomien verstarben 668 Patienten $(5,6 \%)$ noch während des stationären Aufenthaltes.

Der Letalitätsgipfel nach Ersteingriffen lag um den 5. Tag, nach Zweiteingriffen am 2. und nach Dritteingriffen am 1. Tag p.o. 450 Patienten wurden obduziert. Die häufigsten chirurgischen Todesursachen waren Peritonitiden (156 Patienten), die jedoch bei $1 / 3$ der Fälle bereits präoperativ bestanden hatten. 42 Patienten verloren wir an Blutungen und 3 am postoperativen Ileus.

$55 \%$ der Patienten verstarben nicht an chirurgischen Komplikationen. Ein Vergleich mit den gefundenen Risikofaktoren, wie hohes Alter, schlechtes EKG, Diabetes und Adipositas, zeigt, daß nur 4,9\% aller Verstorbenen ohne diese präoperativ bekannten Risikofaktoren waren.

\section{Diskussion zu den Vorträgen 82 bis 101}

Sitzungsleiter: Wir sind damit am Ende unserer Vorträge und kommen zur Diskussion. Wird zu den Ausführungen der einzelnen Herren das Wort gewünscht ? Dann bitte ich darum. 